\title{
BMJ Open Patient experiences and perspectives on hypertension at a major referral hospital in rural southwestern Uganda: a qualitative analysis
}

\author{
Austin Gregory Herbst (D) , ${ }^{1}$ Peter Olds, ${ }^{2}$ Gabriel Nuwagaba, ${ }^{3}$ Samson Okello, ${ }^{3}$ \\ Jessica Haberer ${ }^{1,2,3}$
}

To cite: Herbst AG, Olds $P$, Nuwagaba G, et al. Patient experiences and perspectives on hypertension at a major referral hospital in rural southwestern Uganda: a qualitative analysis. BMJ Open 2021;11:e040650. doi:10.1136/ bmjopen-2020-040650

- Prepublication history and supplemental material for this paper are available online. To view these files, please visit the journal online (http://dx.doi. org/10.1136/bmjopen-2020040650).

$\mathrm{S} 0$ and $\mathrm{JH}$ contributed equally.

Received 18 May 2020

Revised 23 October 2020

Accepted 09 December 2020

Check for updates

(c) Author(s) (or their employer(s)) 2021. Re-use permitted under CC BY-NC. No commercial re-use. See rights and permissions. Published by BMJ.

${ }^{1}$ Harvard Medical School, Boston, Massachusetts, USA

${ }^{2}$ Center for Global Health,

Massachusetts General Hospital, Boston, Massachusetts, USA

${ }^{3}$ Department of Medicine,

Mbarara University of Science

and Technology, Mbarara,

Uganda

\section{Correspondence to} Mr Austin Gregory Herbst; Austin_Herbst@alumni.harvard. edu

\section{ABSTRACT}

Objectives Novel care models are needed to address the large burden of hypertension globally. We aimed to explore how patients in rural Uganda experience and perceive hypertension in order to understand factors that may inform development of a patient-centred care model for hypertension management in this setting.

Design We conducted one-time, in-depth qualitative interviews focusing on participants' experiences and perceptions of the meaning and management of hypertension.

Setting Outpatient clinic at Mbarara Regional Referral Hospital in Uganda.

Participants We enrolled patients who had hypertension and had used antihypertensive medication for at least 1 month. We used purposive sampling to recruit 30 participants with similar representation by gender and by absence or presence of comorbid conditions.

Results Participants had been diagnosed and initiated care at various clinical stages of hypertension, which impacted their understanding of hypertension. Several participants saw hypertension as a chronic disease that can lead to complications if not controlled, while others attributed symptoms typically associated with other diseases to hypertension. Participants described inconsistent access to antihypertensive medications and difficulty with transport to the clinic (time needed and expense) as the major barriers to access to care. Initiation and maintenance of care were facilitated by family support and ready access to health facilities. Many participants identified an understanding of the important lifestyle and dietary changes required to control hypertension.

Conclusions Patients with hypertension in rural Uganda demonstrated a varied understanding and experience with hypertension. Interventions leveraging family support may help with patient education and clinical management. Integration of patient perspectives into the care model, patient-centred care, may serve as a successful model for hypertension and potentially delivery of care for other non-communicable diseases in Uganda and other similar resource-limited settings.

\section{INTRODUCTION}

In 2016, $71 \%$ of global deaths were attributed to non-communicable diseases (NCDs), with
Strengths and limitations of this study

- A major strength of this study is the fact that participants reflect a range of comorbidities and experiences with their hypertension. They were also interviewed at the same clinic and could comment on the same care delivery model for hypertension.

- The rigour of our approach is supported by the achievement of thematic saturation and detailed coding process.

- The study was limited in not including the perspective of family members or healthcare workers. Additionally, the focus on one clinic at a referral hospital means that our findings may not apply to other clinics involving different types of patients or other care delivery models.

- We also did not assess the participants' education level and the role it could have played in their understanding of disease or their medical care.

the largest portion of those attributed to cardiovascular disease. ${ }^{1}$ Hypertension is the leading risk factor for cardiovascular disease, with estimates that it directly leads to as high as $13 \%$ of deaths worldwide. ${ }^{2}$ In Uganda, an estimated one in every four adults has hypertension, and only $10 \%$ are aware of their disease or access care. ${ }^{3}$ Moreover, a prior study of a validated medication adherence tool found that only $15 \%$ of patients met the tool's level of moderate or high adherence to antihypertensive medications. ${ }^{4}$ Given such a high burden and treatment challenges, improved methods of delivering hypertension care are clearly needed. ${ }^{5}$

Patients' experiences and perspectives on hypertension can help shape their treatment. Patient-centred care is a form of health delivery in which multilayered issues, including access to care, disease factors and patient characteristics, are addressed with specific decisions and plans to respond to individual patient's needs and desired outcomes. ${ }^{6}$ This model has been 
shown to be effective at increasing access and adherence to treatment and at improving clinical outcomes. ${ }^{7}$ The WHO considers patient-centred care essential for sustainable NCD management. ${ }^{8}$ Despite the benefits of patientcentred care, few studies have explored how to provide patient-centred hypertension care in low/middle-income countries (LMICs) like Uganda. Of the studies available, most have focused on non-adherence or disease management, ${ }^{9-11}$ and none has used qualitative methods to assess patient-level factors in NCD care in Uganda. However, Siddharthan et al did evaluate a patient-centred tool with educational booklets in the management of heart failure in Kampala, Uganda and noted improved patient engagement with physicians and comfort with self-management of disease. ${ }^{12}$

To inform the development of a patient-centred care model for hypertension management in LMIC settings, we conducted qualitative interviews of patients at a regional referral hospital in rural southwestern Uganda. We specifically sought to understand how patients experience their diagnosis of hypertension, understand the disease and its treatment, access care and manage their hypertension.

\section{METHODS}

\section{Study setting and population}

Care for hypertension in Uganda can be sought at a variety of health centres from Health Centre IV to tertiary care facilities. Specifically, complex cases, often with complications of hypertension, are referred to general, regional or national referral hospitals, which offer increasing degrees of expertise and specialised care, although direct care is available at all facilities. The national level has three levels of hospital: general, regional referral and national referral. Hypertension is typically treated by general practitioners or physicians with training in internal medicine. ${ }^{13}$

Participants for this study were identified from the outpatient hypertension clinic at the Mbarara Regional Referral Hospital, which is located approximately $260 \mathrm{~km}$ southwest of the capital, Kampala. This clinic provides NCD care to over 3000 patients, with an average of 75-120 patients with hypertension seen each week. ${ }^{4}$ Patients are seen on different days of the week based on disease: nephrology and neurology on Monday, hypertension on Tuesday, gastrointestinal on Wednesday, diabetes on Thursday and urology on Friday. Healthcare providers treat patients on a first come, first serve basis. Although medication is provided free of charge, it is often not available due to stock-outs, and patients must buy medications from private pharmacies. When available, primarily calcium channel blockers and thiazide diuretics are used, followed by ACE inhibitors and angiotensin II receptor blockers for those with uncontrolled hypertension.

\section{Patient and public involvement}

Patients and the public were not involved in the design of the study in this report.

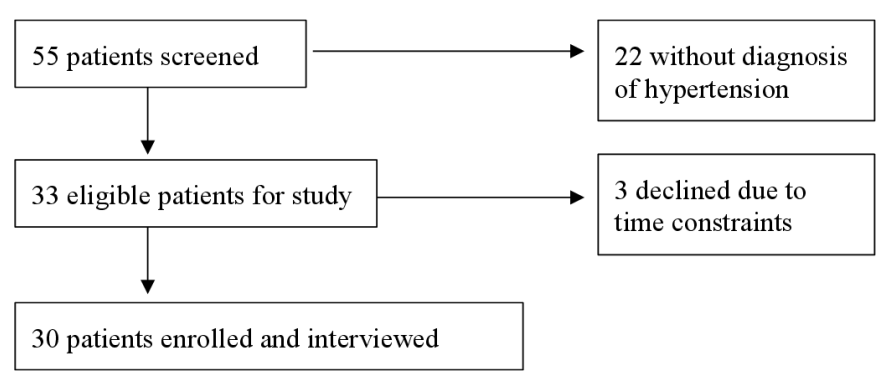

Figure 1 Recruitment and screening of participants.

\section{Sampling and recruitment}

We purposively recruited participants in conjunction with the clinic staff, who used clinic registries and/or personal knowledge to identify potential candidates with a similar ratio of men and women and known comorbid conditions. We aimed for a balanced distribution among participants with hypertension only and hypertension with comorbid diabetes and/or HIV. Potential candidates were approached for written informed consent (online supplemental appendix 1). Recruitment continued until thematic saturation was reached on analysis (figure 1).

In this study, all participants had to have an established diagnosis of hypertension, be 18 years or older, and be able to speak either English or one of the local languages (Runyankole or Rukiga). Pregnant patients were excluded from the study, as pregnancy-related hypertension was considered beyond the scope of the project goals. Patients unable to provide informed consent, including impairment from intoxication or psychosis, were excluded from the study.

\section{Data collection}

We conducted one in-depth, in-person qualitative interview with each participant. The goals of the interviews were to explore the participants' experiences with their diagnosis of hypertension, their understanding of the disease and its treatment, factors involved in access to care and how they approach managing hypertension. Interviews were conducted in an office away from the clinic to ensure privacy and confidentiality. A trained research assistant (GN) fluent in English and Runyankole/Rukiga conducted all interviews following a prespecified interview guide (online supplemental appendix 2). The interview guide was developed by reviewing previous qualitative hypertension studies and discussing key aspects of disease that could be influenced by care delivery models. We used a grounded approach to avoid biasing participant responses. The initial interview guide was tested among members of the team and with two informal interviews of people with hypertension with improvements made in phrasing to increase clarity. The Runyankole/ Rukiga version of the interview guide was back translated to confirm consistency with the English version. Interviews were audio-recorded, translated and transcribed at the same time in English, and reviewed for quality. Data collection lasted approximately 3 months. 


\section{Data analysis}

We analysed qualitative transcripts according to conventional content analysis. ${ }^{14}$ Author AGH reviewed the first five transcripts, analysed content to develop labels, and then created operational definitions and developed a codebook with selected illustrative quotes with significant input from author JH. For quality control, approximately $10 \%$ of interviews were double-coded (authors AGH and PO) and any discrepancies were discussed until consensus was obtained. The codebook was then refined using an iterative process as further interviews were coded. Following completion of the codebook, the remaining transcripts were coded using Dedoose software (V.8.3.11, Los Angeles, California, USA). These codes were used to create a descriptive analysis based on the themes generated from the data and the goals of the study. Quotes were selected to reflect each category and illustrate themes. We used the Standards for Reporting Qualitative Research and Consolidated Criteria for Reporting Qualitative Research checklists when writing our report (online supplemental appendices 3 and 4). ${ }^{15}$

\section{RESULTS}

\section{Participant characteristics}

We screened 55 patients for the study. Of those, 22 were ineligible, primarily because they did not have a documented diagnosis of hypertension. Three additional eligible patients declined participation due to personal time constraints. We thus enrolled and interviewed 30 participants, of whom $20(67 \%)$ were women; the average age was 60 years (SD 14.5 years). Ten (33\%) participants had hypertension only, $10(33 \%)$ had hypertension and diabetes, $1(3 \%)$ had hypertension and HIV, and $9(30 \%)$ had hypertension, diabetes and HIV. Interviews averaged $49 \mathrm{~min}$ (SD $12 \mathrm{~min}$ ) in length.

\section{Overview of qualitative interview results}

We identified four themes representing the experience of participants receiving a hypertension diagnosis and related care over time, starting with (1) participants' initial connection to care, which impacted to how they (2) understood hypertension as a disease. Through the course of their treatment, participants also identified factors that affect (3) access to care and (4) management of hypertension.

\section{Connection to care}

Participants initiated care for hypertension at various stages of disease. Those not yet connected for any general care typically presented when symptomatic; however, some noted that they were asymptomatically diagnosed after screening during an outpatient appointment for another disease or at a local health fair. ${ }^{16}$

Many participants noted that they received a diagnosis of hypertension after a presentation to the hospital with symptoms from another disease, typically either diabetes or a common complication of hypertension, such as myocardial infarction or stroke.

How I came to know that I have hypertension, the same time I was diagnosed with diabetes, I also used to urinate so much. When I narrated the story to someone, how my situation was because I was not having comfortable sleep, he just told me that I am suffering from diabetes, and indeed it was confirmed that I have diabetes and hypertension when I went for treatment. (Male, 86)

I used to be a very sickly person at school in secondary level. I would even collapse, my heart would at times pump so fast... They then brought me to this hospital and diagnosed me with hypertension. They treated me from the outpatients' department, and from there they directed me to the section of the hypertension clinic. They continuously kept checking my hypertension levels... The levels were continuously high. (Female, 28)

We also noted trends among participant characteristics and how they received their diagnosis. For instance, participants with HIV tended to receive their diagnosis of hypertension during a routine clinic visit for HIV care.

I was attending to my usual routine of HIV treatment, and then they took some tests of hypertension and diabetes, and they found out that I had both diseases. My hypertension was very high at 190. From there, I started taking hypertension medication. (Female, 50)

\section{Hypertension as a disease}

When asked what hypertension meant to them as a disease, participants expressed a range of understanding. Most participants were focused on the long-term preventive aspects and cardioprotective nature of hypertension treatment, though how they described their symptomatology depended on the setting of their hypertension diagnosis. Participants who were diagnosed with hypertension either in the outpatient setting during a routine appointment or after symptomatic presentation from hypertension complications tended to prioritise hypertension's long-term risk of stroke and heart disease.

Whenever I come here at the hypertension clinic, I get a chance of meeting people... who were hit by stroke and got paralyzed because of poor management of hypertension. I have to make sure that I always have enough medication of hypertension in order to avoid being hit by stroke and getting paralyzed, too. (Female, 50)

Those diagnosed during a hospitalisation for diabetes or an unrelated illness were more likely to associate hypertension with an array of symptoms. In particular, patients with dual diagnoses of diabetes and hypertension often attributed symptoms of diabetes to hypertension or were unable to differentiate the symptoms of the two diseases. 
P: Hypertension and diabetes are very hard to describe. I actually understand diabetes more than hypertension. The body generally loses all its abilities to do anything. You totally feel so weak. You just feel death is grabbing your entire life. At times you get so dizzy, feel confused in the head, the eyes completely become squinted and start looking at everything in doubles.

I: Is this hypertension or diabetes?

P: I cannot tell the difference of which is which. I take them as one disease. (Female, 71)

Other participants simply stated that they were unsure how to describe hypertension, whereas several participants described hypertension as a 'catch-all' for any unexplained symptoms they may be experiencing.

I don't know, because hypertension is so difficult to explain. Sometime I feel a lot of heat that covers me abruptly, and I start sweating seriously all over my body. But I cannot tell you exactly what hypertension is or how it pains. Not at all. (Female, 72)

Participants generally understood hypertension as a chronic disease. Even with this idea of a chronic disease, they demonstrated resilience and acceptance toward the disease.

It is a very important thing to know your hypertension status because you start treating yourself seriously, maybe you can live longer and do wonderful things in life that you have always wished to do. (Male, 45)

In some, faith played a role in the acceptance.

I just thought about it deeply, and my mind told me, God has a purpose for everything. If this is what he has decided for me, then I will accept it as well. (Female, 71)

\section{Access to care}

Participants noted structural and clinic-based factors that contributed to how and when they accessed care and how it ultimately impacted their approach to management.

\section{Barriers to care}

The cost of hiring transport, both local motorcycles and longer distance taxis, was a significant barrier to care and increased the cost for participants to attend the clinic and make scheduled follow-up appointments.

The medicine I take is very expensive. I also have to come here with a boda boda [motorcycle], which I must pay, and I have to feed as well. (Male, 45)

Participants repeatedly noted financial strain as a major barrier. Decreased available funds, combined with a shortage of the free hypertensive medications supplied by the government at the clinic, led to difficulties purchasing medications with out-of-pocket funds and a decrease in self-reported medication adherence.
P: They always tell me to go and buy, but I do not have the money.

I: How long have you spent without swallowing your hypertension drugs?

P: I have spent about four month, but I am not very sure. I think I last swallowed in March. Whenever they tell me to go and get hypertension from the hospital pharmacy, I do not get anything... They always prescribe the medicine, but the money to buy it is the challenge. (Female, 72)

In addition to accessibility of the clinic impacting care seeking, many participants noted characteristics of the clinic structure that were frustrating and impacted their attitude toward care. Many noted having to arrive at the clinic over 2 hours before the clinic started.

I finished my treatment early today because I woke up so early at around 5:30am. It rained on me, but I wanted to be among the first patient to be treated. In fact, I was the second patient to arrive, and I had to wait...I do not know whether it is because of the many patients or few doctors. Sometimes you wake up early but still you finish your treatment late at around 4:00pm or 5:00pm, when you are very exhausted and tired. (Male, 45)

Several participants compared the ease of accessing treatment and management of HIV to the difficulties of accessing care for hypertension, with some considering it worse than HIV in certain regards.

This disease is more of a risk than HIV. Its rating should be classified as a very dangerous disease. If it is in order, we should be given much more attention than the patients of HIV. These patients of HIV are given counseling, medicine, outreach programs. Really, we should also receive this kind of attention; hypertension is a major risk in families. (Female, 35)

Participants noted a contrast between the availability of care services between different diseases, with more accessible programmes in place for HIV services.

\section{Facilitators to care}

Participants often asked for clinics more easily accessible from their home, either by walking or inexpensive, shortdistance transportation.

How I wish the services were extended nearer to the people in the communities. If they are able to test our hypertension levels in the community, it would be one way of easing treatment for us. (Male, 74)

Additionally, the relationship with health workers was generally positive and encouraged participants to go to the clinic regularly for care.

What I like most is the good relationship we have with these health workers at this hospital. We talk together nicely about health issues. Sometimes, you even 
forget about the challenges you go through. If they were using bad language, people would not be coming to this health facility. (Male, 86)

Participants also expressed the importance of social support, balanced with independence and motivation to manage their own disease. Participants placed particular importance on family and community support, including caregiving, transportation and financial support.

My children are now very helpful. Their father is the one who used to take care of me when he was still alive, but when he passed away, they each gave themselves months when they would buy for me medicine... They usually buy for me the food that I cannot afford, for example fish. They show me so much care and love that keeps me at peace. (Female, 65)

These variable experiences in accessing care shaped the participants' abilities to manage disease and their motivation to seek extensive treatment.

\section{Management of hypertension}

\section{Pharmacological therapies}

Most participants noted that when they had access to medications at home, they always remembered to take their medications and recognised the importance of medication adherence.

No, I have never missed [taking medication]. It is not easy for me to forget. Wherever I go, I have to make sure that I carry my medicine. If I am to spend a night there, I have to make sure that I carry some. It has really never happened. (Male, 72)

Oftentimes, however, participants were unable to pick up medications at the hospital pharmacy, where it is free, due to stock-outs. They were advised to purchase the medication at a private pharmacy. With this added out-of-pocket expense, several participants noted that they would return home without any medications, as they could not afford the costs at private pharmacies.

Money is the most challenging thing while seeking hypertension treatment, especially in buying medicine. For example, the medicine they have prescribed me today is not available here in the hospital, and I have to go and buy it from the drug shops, and I do not have money to buy it. So that becomes a big challenge. (Female, 53)

\section{Alternative therapies}

Participants reported mixed opinions regarding alternative therapies. Many reported not using these therapies because healthcare providers counselled them that the dosing and utility of these therapies were unknown, although some used these therapies in place of medication that they could not afford.
Most of those herbs are so expensive, and I have seen people who stopped swallowing their hypertension or diabetes drugs, and they die so abruptly and painfully. So, I do not trust such medicines. (Female, 53)

I use herbal medicine these days for hypertension because the drugs are very expensive. (Female, 56)

\section{Non-pharmacological therapies}

Participants with hypertension and HIV contrasted the significant non-pharmaceutical management required with NCDs with the primarily pharmaceutical management for HIV care.

HIV is very manageable. I used to do my activities as usual, eat all the foods I want, but with hypertension and diabetes, it's very different. They weaken the body and prevent me from doing all my other activities. You become a real patient and very delicate because you become very selective with the foods... These make life very difficult. (Female, 56)

Many participants noted an understanding of the necessary dietary and physical activity changes that they should make to improve their health and help manage their disease. They noted receiving some form of counselling during the course of their hypertension and diabetes care, listing dietary changes and the importance of exercise.

I was tested and was told I had hypertension. The health workers told me what I should and shouldn't do. In case I was drinking alcohol or smoking I should stop, time reached when I was told to stop eating salt. (Female, 65)

I think it is important because they educate us with how to control it. They say that even if you do not have medicine you can be able to do your exercises very well, do your work very well, your condition may improve somehow. (Male, 64)

Participants endorsed a significant understanding of lifestyle modifications necessary for NCD management, though noted difficulties in maintaining those lifestyle changes. Participants also highlighted finances as a barrier to adherence and alternative therapies as options when they were unable to access their hypertension medications.

\section{DISCUSSION}

In our qualitative study of patients with hypertension in a regional referral hospital in rural Uganda, we found that patients were likely to be connected to care earlier when they had access to health screenings or regular outpatient appointments, presented for another disease, or had easy access to a clinic and family support to encourage care. Participant understanding of hypertension was variable, but closely tied to their symptoms at presentation and/or presence of comorbidities. Financial strain and 


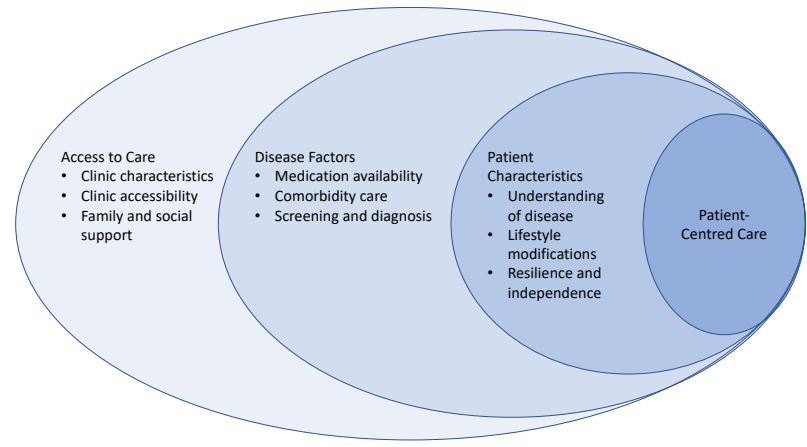

Figure 2 Patient-centred care: factors impacting the patient receipt of care with increasing individualisation of experience.

difficulty travelling to the clinic were among the primary barriers to care, whereas family support and accessible clinics promoted access to care. Additionally, a diagnosis of hypertension was understood to be a chronic, lifelong disease, and participants were generally resilient and eager to continue with disease management through good medication adherence (when possible) and lifestyle changes. All of these components can be used to incorporate patient perspectives into a system that includes generalised patient support, disease integration and patient-level factors as major factors to this patientcentred care model (figure 2).

A major strength of this study is the fact that participants reflect a range of comorbidities and experiences with their hypertension. All participants were seen at the same clinic and could comment on the same care delivery model for hypertension. Furthermore, enrolment continued until thematic saturation was reached. This study is also the first of its kind in trying to understand patient-centred characteristics of hypertension care in Uganda. The study was limited in not including the perspective of family members or healthcare workers. Future studies may benefit from interviewing both caregivers of patients and healthcare providers in order to facilitate a care delivery model that is feasible for both the recipients and deliverers of care. Additionally, the assessment of a single clinic which operates as a referral centre for the region may not be generalisable to other clinics. Also, we did not assess the participants' education level and the role it could have played in their understanding of disease or their medical care.

Considering patient-level factors in healthcare delivery can help ensure adequate management of hypertension and other NCDs by aligning the clinic structure and resources with patient values and beliefs. ${ }^{17}$ Perera et al conducted a similar study to ours in Sri Lanka and also stressed the importance of public education and improved communication between healthcare providers and patients to facilitate care. ${ }^{10}$ Furthermore, Wekesah et al reported similar findings regarding health promotion and awareness in a qualitative study of cardiovascular disease in Nairobi. ${ }^{9}$ Coordinated NCD specialty clinics are currently rare in LMICs; instead, care is divided among specific specialties. Kruk et al suggest redesigning care models in LMICs with a focus on patient and community outreach; this could include integrating care team services and using community healthcare workers to increase access to affordable care ${ }^{18}$ In the Ugandan setting, this model could be used to overcome structural barriers to care or to model the clinic in a way that allows patients to receive care for multiple diseases on 1 day. Routine hypertension care could also be extended into the community, for example, by using health centre II clinics that rely on community health workers. When possible, connecting patients with ways to receive transport or medications at an affordable rate may improve access to care and management of disease. More stable patients may benefit from extended time between follow-up visits to combat expenses for hiring transport to the clinic.

Our study highlights the many ways that patientcentred care can help in improving the delivery of hypertension care in Uganda. As noted, most NCD care in Uganda is provided in specialty clinics, predominantly located at referral centres. Prior groups have integrated NCD care so that patients can receive all their care for chronic diseases at once, thus saving significant time and expense in obtaining care ${ }^{17} 1920$ For example, Peck et al and Wroe et al found integrating NCD care with a patient-centred approach helps to reach patients beyond certain barriers to care and prepare clinics for managing these diseases. ${ }^{19}{ }^{20}$ By integrating NCD care, patients will have decreased travel burden as they receive all their disease care at once. Further, when managing patients' multiple diseases, time can be allotted to customise treatment approaches that fit particular patient preferences and disease burden. Our findings are also supported by studies such as the HOPE-4 programme, which used similar qualitative research in Malaysia and Colombia to incorporate the findings into care models with physicians, non-physician health workers and family members, ultimately determining through a cluster-randomised controlled trial that these interventions can significantly reduce blood pressure and cardiovascular disease. ${ }^{21}$ Similar adaptation of healthcare delivery models should be considered in Uganda.

Patients in general understood the chronicity of the disease and steps required to prevent long-term outcomes, although it may be difficult to follow these steps without support from caregivers and family. Counselling family members alongside patients, including topics related to long-term outcomes of hypertension and what to expect from treatment, may help bridge this gap. By including family and community support in care, patients can better access care and manage their NCDs with appropriate lifestyle management options. ${ }^{22}$ While lifestyle changes may be physically difficult to enact, they can also have significant financial burden, especially for dietary changes. Traditional dietary modification recommendations may be difficult to enact for patients without clear examples of healthy foods that are both affordable and locally available. Increasing community engagement or integrating 
care may facilitate these other changes, and further study toward this is necessary. Participants repeatedly noted that strong social support improved their motivation to manage their disease, suggesting that including family in care would be a welcome approach to combating NCDs. Engagement of family could be particularly important in both the initiation and persistence of lifestyle changes. ${ }^{23}$

Interestingly, participants did not report challenges with medication adherence, although this problem has been widely cited in the literature, including in this setting. ${ }^{42}$ They did note financial constraints to medication adherence, which may be addressed at least in part by more patient-centric care delivery model as described above. Of note, the study did not formally measure adherence and it may have been a larger concern than reported in the interviews due to social desirability or recall bias. ${ }^{25}$

\section{CONCLUSION}

In our study of patients with hypertension in southwestern Uganda, experience varied considerably regarding receiving a diagnosis and comorbid conditions. Structural barriers, including clinic accessibility and availability of medications, play major limitations in disease care. Patient-centred care in this setting should seek to reduce these barriers through integrating NCD care in the community and building up family support in disease education and management. By including the patient perspectives in models of care, patient-centred care may be able to improve healthcare delivery and outcomes.

Acknowledgements The authors would like to acknowledge the MRRH clinic; Mbarara University of Science and Technology for research space; and all participants for their time.

Contributors Authors AGH, JH, PO and SO conceived the study and protocol. Authors AGH, SO and GN implemented the study; and author GN conducted and transcribed the interviews. AGH wrote the initial draft of the paper, which was reviewed, edited and approved by all authors.

Funding Support for this research was provided by Harvard Medical School. JH was supported by K24MH114732. S0 is supported by NIH K43TW010715. AGH was supported by the Harvard Medical School Traveling Fellowship.

Competing interests $\mathrm{JH}$ reports grants from $\mathrm{NIH}$, during the conduct of the study; personal fees from Merck, outside the submitted work.

Patient consent for publication Not required.

Ethics approval The study was approved by the Mbarara University of Science and Technology Faculty of Medicine Research Ethics Committee; the Partners Health Care Human Research Committee; and the Harvard Medical School and Harvard School of Dental Medicine Institutional Review Board, via Reliance. We additionally received approval from the Uganda National Council on Science and Technology. All participants provided written informed consent or thumbprint in the case of illiteracy (see online supplemental appendix 5 for full protocol).

Provenance and peer review Not commissioned; externally peer reviewed.

Data availability statement Data are available upon reasonable request. Participant data that underlie the results reported in this paper are available following the paper's publication and under appropriate data sharing agreements. We will also provide the study protocol, analysis plan and language of the consent forms. Data are available for researchers who provide a methodologically sound proposal, which should be directed to Austin_Herbst@alumni.harvard.edu.

Supplemental material This content has been supplied by the author(s). It has not been vetted by BMJ Publishing Group Limited (BMJ) and may not have been peer-reviewed. Any opinions or recommendations discussed are solely those of the author(s) and are not endorsed by BMJ. BMJ disclaims all liability and responsibility arising from any reliance placed on the content. Where the content includes any translated material, BMJ does not warrant the accuracy and reliability of the translations (including but not limited to local regulations, clinical guidelines, terminology, drug names and drug dosages), and is not responsible for any error and/or omissions arising from translation and adaptation or otherwise.

Open access This is an open access article distributed in accordance with the Creative Commons Attribution Non Commercial (CC BY-NC 4.0) license, which permits others to distribute, remix, adapt, build upon this work non-commercially, and license their derivative works on different terms, provided the original work is properly cited, appropriate credit is given, any changes made indicated, and the use is non-commercial. See: http://creativecommons.org/licenses/by-nc/4.0/.

\section{ORCID iD}

Austin Gregory Herbst http://orcid.org/0000-0002-6705-3727

\section{REFERENCES}

1 WHO. Global Health Observatory (GHO) data NCD mortality and morbidity. World Health Organization, 2018. Available: http://www. who.int/gho/ncd/mortality_morbidity/en/

2 Arima H, Barzi F, Chalmers J. Mortality patterns in hypertension. $J$ Hypertens 2011;29 Suppl 1:S3-7.

3 Guwatudde D, Mutungi G, Wesonga R, et al. The epidemiology of hypertension in Uganda: findings from the National noncommunicable diseases risk factor survey. PLoS One 2015;10:e0138991.

4 Okello S, Nasasira B, Muiru ANW, et al. Validity and reliability of a self-reported measure of antihypertensive medication adherence in Uganda. PLoS One 2016;11:e0158499.

5 World Health Organization. Uganda non-communicable diseases Factsheet. World Heal Organ - Noncommunicable Dis Ctry Profiles 2018;2018.

6 NEJM Catalyst. What Is Patient-Centered Care? NEJM Catal Innov Care Deliv, 2017. Available: https://catalyst.nejm.org/what-is-patientcentered-care/

7 De Man J, Mayega RW, Sarkar N. Patient-Centered care and PeopleCentered health systems in sub-Saharan Africa: why so little of something so badly needed? Int J Pers Cent Med 2016;6.

8 Package of Essential Noncommunicable (PEN). Disease interventions for primary health care in low-resource settings

9 Wekesah FM, Kyobutungi C, Grobbee DE, et al. Understanding of and perceptions towards cardiovascular diseases and their risk factors: a qualitative study among residents of urban informal settings in Nairobi. BMJ Open 2019;9:e026852.

10 Perera M, de Silva CK, Tavajoh S, et al. Patient perspectives on hypertension management in health system of Sri Lanka: a qualitative study. BMJ Open 2019;9:e031773.

11 Ashoorkhani M, Majdzadeh R, Gholami J, et al. Understanding NonAdherence to treatment in hypertension: a qualitative study. Int $J$ Community Based Nurs Midwifery 2018;6:314-23.

12 Siddharthan T, Rabin T, Canavan ME, et al. Implementation of patient-centered education for Chronic-Disease management in Uganda: an effectiveness study. PLoS One 2016;11:e0166411.

13 Primary health care systems (PRIMASYS): case study from Uganda, abridged version. Geneva, 2017. Available: http://apps.who.int/ bookorders

14 Patton MQ, \&amp QR;amp, Methods E. Thousand oaks. 3rd ed. CA: SAGE Publications, 2002.

15 O'Brien BC, Harris IB, Beckman TJ, et al. Standards for reporting qualitative research: a synthesis of recommendations. Acad Med 2014;89:1245-51.

16 Outcomes H. Progressive entrepreneurship and networks. Available: http://ghcuganda.org/our-work/research-innovation/hopenet/

17 Geldsetzer P, Ortblad K, Bärnighausen T. The efficiency of chronic disease care in sub-Saharan Africa. BMC Med 2016;14:127.

18 Kruk ME, Nigenda G, Knaul FM. Redesigning primary care to tackle the global epidemic of noncommunicable disease. Am J Public Health 2015;105:431-7.

19 Peck R, Mghamba J, Vanobberghen F, et al. Preparedness of Tanzanian health facilities for outpatient primary care of hypertension and diabetes: a cross-sectional survey. Lancet Glob Health 2014;2:e285-92.

20 Wroe EB, Kalanga N, Mailosi B, et al. Leveraging HIV platforms to work toward comprehensive primary care in rural Malawi: the integrated chronic care clinic. Healthc 2015;3:270-6.

21 Schwalm J-D, McCready T, Lopez-Jaramillo P, et al. A communitybased comprehensive intervention to reduce cardiovascular risk in 
hypertension (hope 4): a cluster-randomised controlled trial. Lancet 2019;394:1231-42.

22 BeLue R. The role of family in non-communicable disease prevention in sub-Saharan Africa. Glob Health Promot 2017;24:71-4.

23 Lawrence M, Kerr S, Watson $\mathrm{H}$, et al. An exploration of lifestyle beliefs and lifestyle behaviour following stroke: findings from a focus group study of patients and family members. BMC Fam Pract 2010;11:97.
24 Mugwano I, Kaddumukasa M, Mugenyi L, et al. Poor drug adherence and lack of awareness of hypertension among hypertensive stroke patients in Kampala, Uganda: a cross sectional study. BMC Res Notes 2016:9:3.

25 Choo PW, Rand CS, Inui TS, et al. Validation of patient reports, automated pharmacy records, and pill counts with electronic monitoring of adherence to antihypertensive therapy. Med Care 1999;37:846-57. 